\title{
A knapsack problem with mixed distributional weight
}

\author{
Akpan, N. $\mathrm{P}^{1}$ and Etuk, E. $\mathrm{H}^{2}$ \\ ${ }^{1}$ Department of Mathematics and Statistics, University of Port Harcourt, Port Harcourt, Rivers \\ State, Nigeria \\ ${ }^{2}$ Department of Mathematics \& Computer Science Rivers State University of Science and \\ Technology, Port Harcourt, Rivers State, Nigeria
}

\begin{abstract}
In this paper we present a knapsack problem whose weight parameter is a mixture of two known distributions (Exponential and Gamma). This problem gives room for the overflowed items which perhaps will help in minimizing the penalty due to the loss of goodwill. An algebraic model is proposed for solving the problem. The behaviors of the mixture of these two distributions are also presented graphically.
\end{abstract}

Key words: Knapsack Problem, Mixed Distribution, Mill, Stochastic, Overflow, Exponential Distribution, Gamma Distribution

\section{INTRODUCTION}

Our study of the stochastic knapsack problem was initially motivated by the series of activities that take place in a firm that processes several products such as palm fruits for palm oil, cassava grinding for garri, palm kernel cracking, etc. where the arrival of customers is stochastic and the bill for the customers is directly proportional to the quantity (weight) of items brought by them. It is worth stating that there is one major machine with different attachments that is used for these operations which is manned by an operator. Here, we wish to extend the work by considering a situation in which the weight of the items which signifies the quantity of these items is assumed to follow a combination of two distributions. Although we assume that this item (the weight) follows a combination of two distributions, it is obvious that we cannot predict the number of customers that will come to the mill in each day for their business neither can we predict the quantity of goods (items) they will bring.

However, literature has shown that much has been done in stochastic knapsack problem especially when the weight is random or the capacity is deterministic. In this paper we will deviate from that trend by considering a situation where the weight is a mixture of two known distributions as earlier mentioned, and the mixture is of the multiplicative model. The weight is a mixture of Exponential and Gamma distribution. Meanwhile, we must state that the items are accepted on the basis that the costumer must accept the bill given to him/her by the operator given the quantity (weight) of the items brought and the available space (capacity). Hence the expansion of our model will consider those items (weight) which the customers have accepted to pay the required amount for the services but the capacity is exceeded (i.e the overflow). Following the idea of Cohn and Barnhart (1998) we intend to maximize the expected value of the chosen objects minus the penalty of exceeding capacity.

Researchers have worked on the area of stochastic knapsack problem to achieve their goals. For instance Goel and Indyk (1999) considered the case where the item weights in a particular instance are not fixed constants but random variables $W_{i}$ with a given distribution function $F_{i}$. His model was completely different in the sense that, the constraint of knapsack problem turns into a probability condition with a given overflow probability $\pi$. That is the problem

$$
\begin{aligned}
& \text { Maximize } \sum_{i=1}^{n} p_{i} x_{i} \\
& \text { S.t } \quad \sum_{i=1}^{n} w_{i} x_{i} \leq c \\
& x_{i} \in\{0,1\} \quad i=1, \ldots, n
\end{aligned}
$$

Becomes

$$
\text { Maximize } \sum_{i=1}^{n} p_{i} x_{i}
$$


S.t $\quad P\left(\sum_{i=1}^{n} w_{i} x_{i} \leq c\right) \geq 1-\pi \quad \ldots \ldots \ldots$.eqn (1a)

A deterministic algorithm was used to decide on which items to pack based on the given constant profit values $p_{i}$ and the distributional function $F_{i}$, respectively the expected values $E\left[W_{j}\right\rfloor$, but without knowing the actual realization of $W_{i}$.

This same stochastic model of random weights represented by random variables $w_{i}$ was considered in the numerical experiments of the more general paper of Kleywegt et al (2001). Instead of maximizing the profit under an overflow probability as in equation (1a) above, Kleywegt et al (2001) suggested that the possible overflow in the objective function is given by the following combination of profit values and expected value:

Maximize $\sum_{i=1}^{n} P_{i} x_{i}-q^{*} E\left[\operatorname{Max}\left\{0, w_{i} x_{i}-c\right\}\right]$

Where $q$ is a constant factor for each unit of overflow; see Kellera et al 2004.

Cohn \& Barnhart (1998) slightly extended the basic model of random weight and constant profit in a way that, the weight are still random variables $W_{i}$ with a given distribution function $F_{i}$. Also, the profits of an item $i$ are now given by the weight multiplied by a constant scaling factor $S_{i}$. Hence the profits are dependent on the same distribution as the weights. They considered a case where the $F_{i}$ are independent normal distribution functions, but not necessarily identical. The objective function considered here is almost similar to that of Kleywegt et al in equation (2) above except that the expectation must be taken also over the profits as

$$
\begin{gathered}
\text { Maximize } E\left[\sum_{i=1}^{n} s_{i} W_{i} x_{i}-q \cdot \max \left\{0, \sum_{i=1}^{n} W_{i} x_{i}-c\right\}\right]= \\
=\sum_{i=1}^{n} s_{i} E\left[W_{i}\right] x_{i}-q \cdot E\left[\operatorname{Max}\left\{0, \sum_{i=1}^{n} W_{i} x_{i}-c\right\}\right]
\end{gathered}
$$

According to Kellerer et al (2004), another type of stochastic knapsack problem with reversed roles of constants and random values was discussed by a number of authors starting (to the best of knowledge) with the paper by Steinberg \& Parks(
1979) and continued in the work of Sniedovich (1980),(1981), Carraway et al (1993), Morita et al (1989), Henig(1990), Morton \& Wood(1998), to mention a few.

Willmot (1986) stated that the distribution of total claims payable by an insurer is considered when the frequency of the claims is a mixed poisson random variable. He added that, mixed poisson distributions often have desirable properties for modeling claim frequencies. He showed how in many cases of claims density can be evaluated numerically using simple recursive formular (discrete and continuous). Liu et al (2002) proposed a Poisson-Gumbel mixed compound distribution, one of a particular form of Bivariate Compound Extreme Value Distribution, and gave an example of its application by using it to compute the joint distribution of wind velocities and wave heights and to estimate the 100-year return level, based on the 20-year wind and wave data in the East China sea.

Thamerus(1996) fitted a finite mixture distribution to a variable subject to Heteroscadastic measurement error. In his work, he considered the case where a latent variable $X$ cannot be observed directly and instead a variable $W=X+U$ with an Heteroscedastic measurement error $U$ is observed. It is assumed that the distribution of the true variable $X$ is a mixture of normals and a type of EM algorithm was applied to find approximate maximum likelihood estimates of the distribution parameters of $X$. De Carlo \& Lawrence(2002) worked on signal detection theory with finite mixture distributions: theoretical developments with applications to recognition memory.

Arana and Leon (2004) considered the performance of a model of mixture normal distributions for dichotomous choice contingent valuation data, which allows the researcher to consider unobserved heterogeneity across the sample. The model is flexible and approaches a semi parametric model, since any empirical distribution can be represented by augmenting the number of distributions. The proposed model was compared with other semiparametric and parametric approaches using Monte Carlo simulation, under alternative assumptions regarding heteroscedasticity and heterogeneity in sample observations. It was found that the mixture normal model reduces bias and improves performance with respect to an alternative semi- 
Am. J. Sci. Ind. Res., 2011, 2(6): 865-870

parametric model, particularly when the sample is characterized by heterogeneous preferences.

Johnson and Kotz (1969) worked on the mixture of Poisson over the Beta Distribution. The distribution was given by

$$
\begin{aligned}
& d U(x)=\frac{\beta(\mu-x)^{\beta-1}}{\mu^{\beta}} d x \quad 0<x<\mu \\
& \text { Thus } \\
& P_{n}=\beta \int_{0}^{\mu} \frac{\lambda^{n} e^{-\lambda}}{n !} * \frac{(\mu-\lambda)^{\beta-1}}{\mu^{\beta}} d \lambda \\
& =\frac{\mu^{n}}{n !} \Gamma(\beta+1) \sum_{k=0}^{\infty} \frac{\Gamma(n+k+1)}{\Gamma(\beta+n+k+1)} * \frac{(-\mu)^{k}}{k^{1}}, \quad \mathrm{n}=0,1,2, \ldots
\end{aligned}
$$

A more general Beta mixture was discussed by Willmot and Panjer (1985). However, the above mixture is useful in obtaining results for more general severity distributions for certain choices of $\beta$.

According to Buckley et al (2003), the gaussian mixture of distributions has been used before in the field of finance, mostly in the univariate guise for the estimation of value at risk. Hull \& White (1998) in their work, employed the univariate mixture of normal distributions on the model he developed for estimating variance at risk. More recently Guassian mixture models have been used to model future markets for portfolio risk management, see Labidi \& Thierry (2000). Also, Mc Neil et al (2001) use it for credit risk. Lee et al (2010) in their work on Direct deconvolution density estimation of a mixture distribution motivated by mutation effects distribution focus on the case that the distribution of the target variable $X$ is a mixture of pointmass and a continuous distribution. They assumed $X_{d}$ to be the degenerate component of $X$ and $X_{c}$ to be the continuous component. Then represented $X$ as $X=\left\{\begin{array}{l}X_{d} \text { with probability } P \\ X_{c} \text { with probability } 1-P\end{array}\right.$

Where $P$ is the unknown mixing probability. They Supposed that the location of $X_{d}$ is known, that is, $P\left(X_{d}=a\right)=1$ for some known constant a. Then the generalized density, Cuevas \& Walter (1992) of $X$, say $f(x)$, can be expressed as

$$
f_{x}(x)=P \delta_{a}(x)+(1-P) f_{c}(x)
$$

Where $\delta_{a}$ denotes the dirac delta at $\mathbf{a}$, and $f_{c}$ is the density of $X_{c}$

\section{ASSUMPTIONS}

1) The items arrive independently and randomly

2) The weight of each item is not known until its arrival

3) The profit or reward for each item is directly proportional to its weight

4) All items have the same distribution but their weight and reward vary.

Mixed distribution: Dimitris \& Evdokia (2005) observed that a mixture of distributions have been widely used for modeling observed situations whose various characteristics as reflected by the data differ from those that would be anticipated under the simple component distribution. He added that a probability distribution is said to be a mixture distribution if its distribution function $F($.$) can be$ written in the form

$$
F(.)=\int_{\Theta} F(. / \lambda) d G(\lambda)
$$

Where $F($.$) denotes the distribution function of the$ component densities considered to be indexed by a parameter $\lambda$ With distribution function $G(\lambda), \lambda \in \Theta$. This definition can also be expressed in terms of probability density functions thus;

$$
f(x)=\int_{\Theta} f(x / \lambda) g_{\lambda}(\lambda) d \lambda
$$

However Mood et al(2006) in their own way stated that, if $f_{0}(),. f_{1}(),. \ldots, f_{n}(),. \ldots$ is a sequence of density functions which are either all discrete density functions or all probability density functions which may or may not depend on parameters and $P_{0}, P_{1,}$. ., $P_{n}$, is a sequence of parameters satisfying $P_{i} \geq 0$ and $\sum_{i=0}^{\infty} P i=1$, then $\sum_{i=0}^{\infty} P i f i(x)$ is a density function, which is sometimes called a contagion or mixture. For example if $\mathrm{f}_{0}(\mathrm{x})=\Phi \mu_{0}, \delta_{0}^{2}(x)$ (a normal with mean $\mu_{0}$ and variance $\left.\delta_{0}^{2}\right)$ and $\mathrm{f}_{1}(\mathrm{x})=\Phi \mu_{1}, \delta_{1}^{2}(x)$ then

$$
\begin{aligned}
& P_{0} \Phi \mu_{0}, \delta_{0}^{2}(x)+P_{1} \Phi \mu_{1} \delta_{1}^{2}(x)= \\
& (1-P) \frac{1}{\sqrt{2 \pi \delta_{0}^{2}}} \ell^{-\frac{1}{2}\left[\left(x-\mu_{0}\right) / \delta_{0}\right]^{2}}+P \frac{1}{\sqrt{2 \pi \delta_{1}^{2}}} \ell^{-\frac{1}{2}\left[\left(x-\mu_{1}\right) / \delta_{1}\right]^{2}}
\end{aligned}
$$

Where $\mathrm{P}_{1}=\mathrm{P}$ and $\mathrm{P}_{0}=1-\mathrm{P}$, is a mixture of two normal densities. 
He further stated the extension of a mixed distribution as; Let $\{f(x ; \theta)\}$ be a family of density functions parameterized or indexed by $\theta$. Let the totality of values that the parameter $\theta$ can assume be denoted by $\Theta$. If $\Theta$ is an interval (possibly infinite) and $g(\theta)$ is a probability density function which is zero for all arguments not in $\Theta$, then

$$
\int_{\Theta} f(x ; \theta) g(\theta) d \theta
$$

Is a density function, called a mixed distribution.

\section{A mixture of Exponential and Gamma Distribution:}

$$
\text { Let } \begin{aligned}
& f(x ; \theta)=\theta \ell^{-\theta x} ; \quad x>0 \\
& g(\theta)=\frac{\lambda^{r}}{\Gamma(r)} \theta^{r-1} \ell^{-\lambda \theta}
\end{aligned}
$$

Then $\int_{0}^{\infty} f(x ; \theta) g(\theta) d \theta=\int_{0}^{\infty} \frac{\lambda^{r}}{\Gamma(r)} \theta^{r-1} \ell^{-\lambda \theta} \theta \ell^{-\theta x} d \theta$

$=\int_{0}^{\infty} \frac{\lambda^{r}}{\Gamma(r)} \theta^{r+1-1} \ell^{-(\lambda+x) \theta} d \theta$

$=\frac{\lambda^{r}}{\Gamma(r)} * \frac{\Gamma(r+1)}{\Gamma(r+1)} * \frac{(\lambda+x)^{r+1}}{(\lambda+x)^{r+1}} \int_{0}^{\infty} \theta^{(r+1)-1} \ell^{-(\lambda+x) \theta} d \theta$

$=\frac{\lambda^{r} \Gamma(r+1)}{\Gamma(r)(\lambda+x)^{(r+1)}} \int_{0}^{\infty} \frac{(\lambda+x)^{(r+1)}}{\Gamma(r+1)} \theta^{(r+1)-1} \ell^{-(\lambda+x) \theta} d \theta$

$=\frac{\lambda^{r} \Gamma(r+1)}{\Gamma(r)(\lambda+x)^{(r+1)}}=\frac{\lambda^{r} r}{(\lambda+x)^{r+1}}$

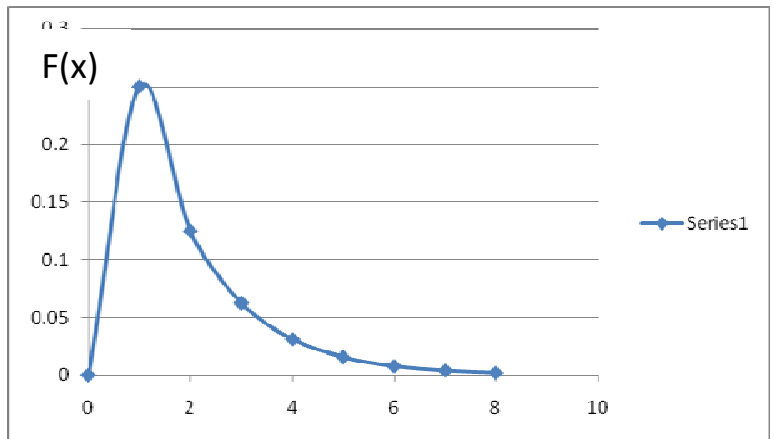

A mixture of exponential and gamma Distribution with $\lambda=r=x$ and $0 \leq x \leq 8$

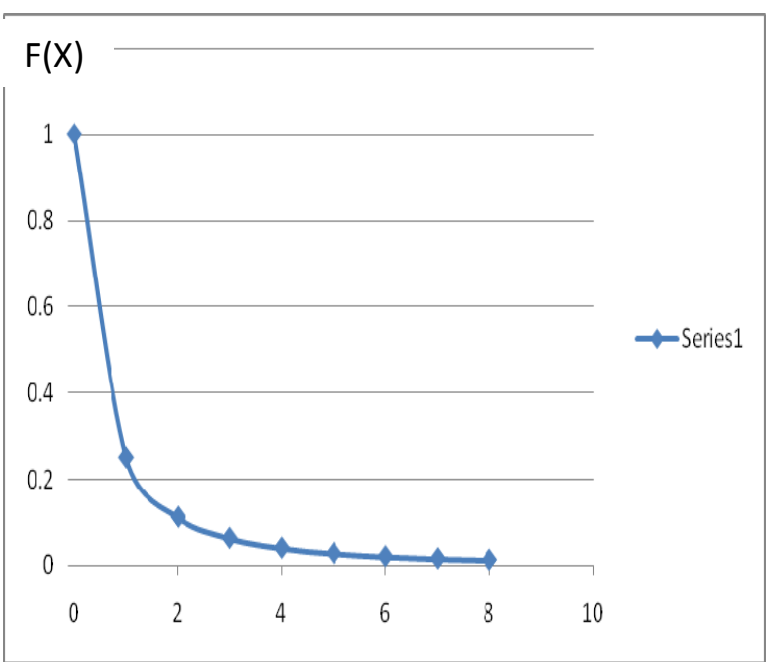

A mixture of exponential and gamma Distribution with $\lambda=r=1$ and $0 \leq x \leq 8$

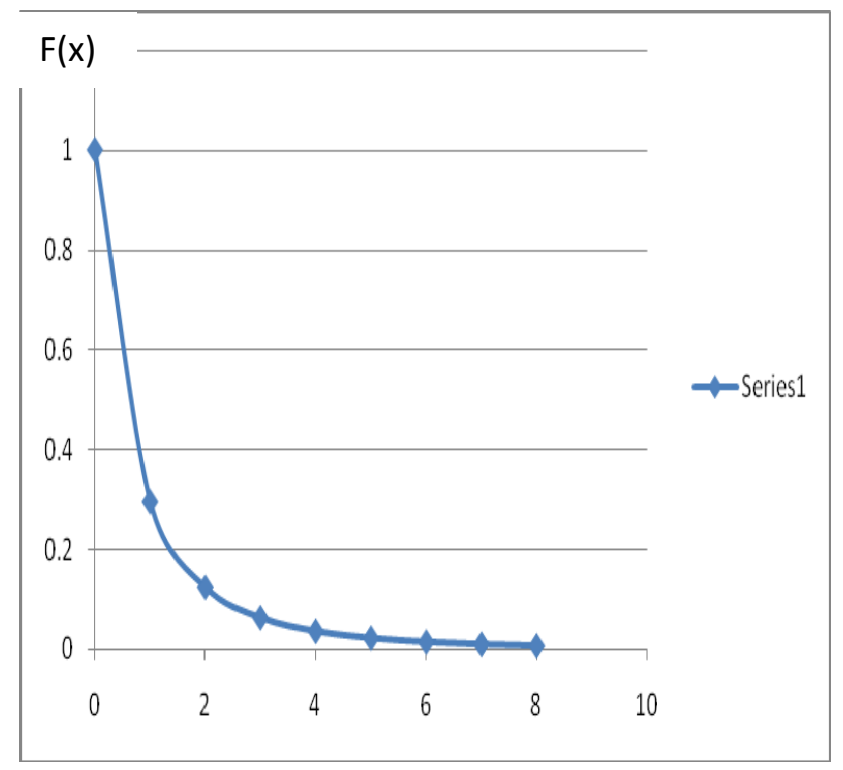

A mixture of exponential and gamma Distribution with $\lambda=r=2$ and $0 \leq x \leq 8$ 


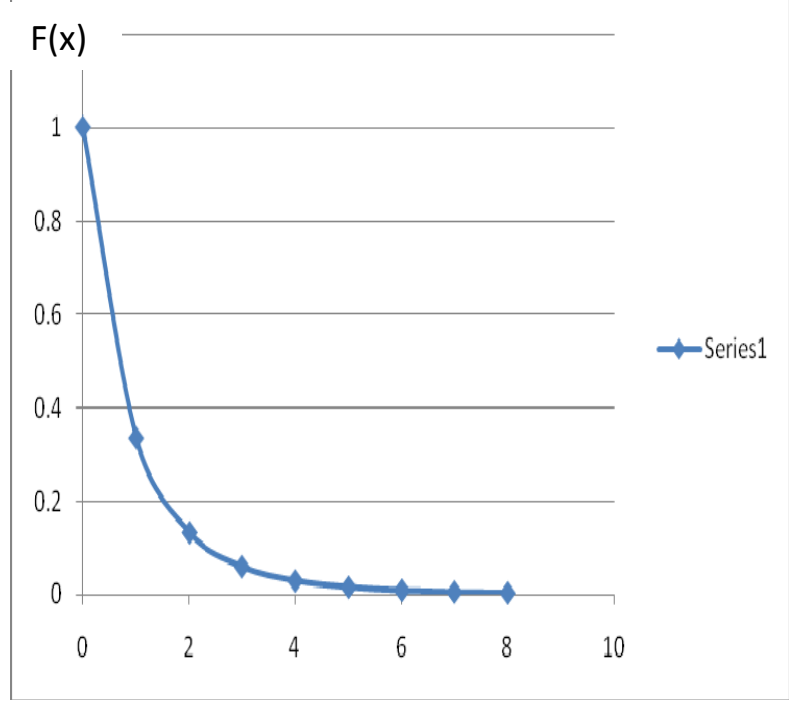

A mixture of exponential and gamma Distribution with $\lambda=r=5$ and $0 \leq x \leq 8$

The above graphs show the behavioral pattern as the parameters of the mixed distribution varies. For instance, when $\lambda=r=x$, its output is a peaked curve that skewed to the right and whereas the other ones follow a similar pattern (i.e when $\lambda=r=1, \lambda=r=2$, and $\lambda=r=5$ )with $0 \leq x \leq 8$; a decreasing function graph.

Model formulation: We further assume that each item $I$ is independent, and it arrival time is also independent of each other and is a random event. Also the capacity of the knapsack is known already. Meanwhile, the leftover that cannot be accepted each day constitute what we termed the capacity overflow which often lead to the loss of goodwill by the customers will be assessed by a penalty of $\phi$ per item. As such our model will embrace this penalty. This consideration is because, while the firm is maximizing the profit with respect to the selected weight, it is good to note that she must minimize her inability to satisfy the customers which implies minimizing the rate of penalty to be paid. Thus, our proposed model is:

$$
\begin{aligned}
& \text { Maximize } \sum_{i=1}^{B} P_{i} x_{i}-\phi \int_{B+1}^{\infty} f(w) y(w) d w \cdots \ldots . .(4) \\
& \text { S.t } \sum_{i=1}^{n} w_{i} x_{i} \leq C+y(w)
\end{aligned}
$$

Where $P_{i}$ is the profit of item $i$ $x_{i}$ is the binary decision variable

$\phi$ is the penalty per weight of the overflow whom the customer has agreed to pay the bill.

$w_{i}$ is the weight of item $i$

$\mathrm{C}$ is the capacity of the knapsack

$B$ is the nth item that filled the knapsack

$y(w)$ is the expected cost of the overflowed weight $f(w)$ is the density function of the weight

From equation (4) we can have

$$
\begin{aligned}
\text { Maximize } & \sum_{i=1}^{B} P_{i} x_{i}-\phi \int_{B+1}^{\infty} \frac{\lambda^{r} r}{(\lambda+x)^{r+1}} y(w) d w \\
\mathbf{x} & \text { S.t } \sum_{i=1}^{n} w_{i} x_{i} \leq C+y(w)
\end{aligned}
$$

\section{CONCLUSION}

In this paper, we have mixed two continuous distribution using a multiplicative model. The graphical presentation showing the behavior of the variation of the parameters has been made. We have also proposed a mathematical model for a stochastic knapsack problem where the weights of the items follow a mixture of two known distribution; Exponential and Gamma.

\section{REFERENCES}

ARANA, J. E. \& LEON,C. J. (2004). Flexible mixture distribution modeling of dichotomous choice contingent valuation wiyh Heterogeneity. Econometric society, North American Summer meeting with number 568

BUCKLEY, I.,COMEZANA, G., DJERROUD, B. \& SECO, L.(2003): Portfolio Optimization when asset returns have the Gaussian Mixture Distribution. Centre for Quantitative finance. Imperial College, London, $\mathrm{SW}_{7}$ 2BX..UK

CARRAWAY,R.I, SCHMIDT \& WEATHERFORD,L.R (1993): An algorithm for maximizing target achievement in the stochastic knapsack problem with normal returns. Naval Research Logistics. 40:161173

COHN, A. \& BARNHART, C. (1998): The Stochastic Knapsack Problem with Random Weights: A heuristic approach to robust transportation planning, proceedings of the Triennial symposium on Transportation Analysis (TRISTAN III)

CUEVAS, A \& WALTER, G. (1992)On estimation of generalized densities, Cummum Stat.- Theory Methods 21, pp 1807-1821 
DE CARLO, LAWRENCE T. (2002), Signal Detection theory with finite mixture distribution: Theoretical developments with applications to Recognition memory. Psychological Review Vol.109,No.4, 710721

DIMITRIS K. \& EVDOKIA, X. (2005): Mixed poisson Distribution. International Statistical Institute.

GOEL,A., \& INDYK,P.(1999): Stochastic load balancing and related problems. In: Proceedings of the $40^{\text {th }}$ Annual Symposium on Foundations of Computer Science, pp 579-586

HENIG, M. I (1990): Risk criteria in a stochastic knapsack problem. Operations Research, 38:820-825

HULL, J. \& WHITE A. (1998) Value at Risk when daily changes in market variables are not normally distributed, Journal of Derivatives 5, no.3, 9-19

JOHNSON, N.L. \& KOTZ, S. (1969), Distributions in statistics Discrete Distributions. John Wiley, New York

KELLERER, H., PFERSCHY, U. \& PISINGER, D.(2004) Knapsack problems. ISBN 3-540-40286-1 SpringerVerlag Berlin Heidelberg New York.

KLEYWEGT,A. J, SHAPIRO,A. \& HOMEM-DE-MELLO, T.(2001). The sample average approximation method for stochastic discrete optimization. SIAM Journal on Optimization, 12:479-502

LABIDI CHIRAZ \& THIERRY AN (2000) Revisiting the finite mixture of guassian distributions with applications to future markets, Computing in Economics and Finance, No.67

LEE, M., SHEN, H., BURCH, C., \& MARRON , J. S. (2010), Direct Deconvolution density estimation of a mixture distribution motivated by mutation effect distribution. Journal of non parametric statistics Vol.22 No. 1, 1-22
LIU D., WEN S. \& WANG L. (2002) Chinese Science bulletin Vol 47 No 22

MC NEIL, A. J., NYFELER M., FREY, R., (2001), Copullas and credit models, RISK, 111-114

MOOD, A.,GRAYBILL,F.,\& BOES, D. (2006): Introduction to the Theory of Statistics, $3^{\text {rd }}$ Edition, Mc Graw-Hill

MORITA, H.,ISHII, H.,\& NISHIDA, T.(1989):Stochastic Linear knapsack programming problem and its application to portfolio selection problem. European Journal of Operational Research, 40:329-336

MORTON, D.P \& WOOD,R.K (1998): On a stochastic knapsack problem and generalizations. Advances in computational and Stochastic Optimization, Logic Programming and Heuristic search, ed. Woodruff, D.L. pages $149-169$

SNIEDOVICH, M. (1980): Preference order stochastic knapsack problems: methodological issues. Journal of Operations Research Society 31:1025-1032

SNIEDOVICH, M. (1981): Some comments on preference order dynamic programming models. Journal of Mathematical Analysis and Applications. 79:489-501

STEINBERG,E. \& PARKS, M. S.(1979): A preference order dynamic program for a knapsack problem with stochastic rewards. Journal of the operational Research Society, 30: 141-147

THAMERUS MARCUS (1996), Fitting a finite mixture distribution to a variable subject to heteroscedastic measurement error. Sonder for schungsbereich 386, paper 48

WILLMOT G. (1986) ; ASTIN Bulletin Vol 16,S59 - S79. www.casact.org/library/vol16s/s59pdf

WILLMOT,G.E \& PANJER,H.H (1985): Difference Equation Approaches in evaluation of compound distribution to appear. 